\title{
Practical Universalism and Multiple Social Categorization: Can Different Social Biases Counterbalance Each Other?
}

\author{
Alexander V. Shkurko ${ }^{a^{*}}$ \\ ${ }^{a}$ Independent Researcher, Ulyanovsk, Russia \\ ${ }^{\star}$ Corresponding author. E-mail: khanovey@protonmail.com
}

Background. Normative universalism involves making evaluations and decisions according to a universal rule, irrespective of one's affiliation and relations with other people. Social categorization is the main cognitive mechanism underlying deviations from universalism. When there are several salient alternative social identities, there is a possibility of counterbalancing effects among different social biases, leading to unbiased decisions or judgments (i.e., practical universalism).

Objective. The present study investigates whether multiple categorization can induce alternative social biases, which counterbalance each other and produce universalistic solutions at both the individual and group levels.

Design. A socially heterogeneous sample of Russian participants $(\mathrm{N}=300)$ made a series of binary choices in a hypothetical situation posing two social alternatives, each of which was presented as a set of social categories unrelated to the task: country, gender, age, and sector of employment.

Results. When faced with a series of choices involving multiple social categorization, the participants tended to pursue different types of biased strategies. The most frequent were country ingroup preference (31.7\%) and low status aversion (17.7\%). Practical universalism was identified in $2 \%$ of cases. Group-level results showed strong ingroup preference and high-status preference, which are two independent sources of bias. At the same time, the diversity of individual strategies allowed the participants to identify conditions (specific combinations of social attributes) under which the biases counterbalanced each other and resulted in universalistic solutions.

Conclusion. Individuals respond strategically to multiple categorization. Universalistic strategies are seldom applied at the individual level, but the diversity of individual strategies provides opportunities for universalistic solutions at the group level.

Keywords: universalism; particularism; multiple social categorization; social bias; social status 


\section{Introduction}

Universalism is a normative idea, which calls for making judgments, evaluations, and decisions according to a universal rule, irrespective of one's affiliation and relations with other people (Parsons, 1937; Trompenaars \& Hampden-Turner, 2012; Ma \& McLean Parks, 2007). Universalism means that when some of a person's social attributes are not relevant to a given situation or task, such information about them should be ignored during evaluations or decision-making. Universalistic reactions imply that a rule be applied in the same way irrespective of a target's social attributes, which are not relevant for the task or situation.

Particularistic reactions allow exceptions from a universal rule depending on a target's social characteristics or personal relations with the actor. A typical example is a police officer stopping a car driver for speeding. According to a universalistic approach, the driver should be fined no matter who he/she is. However, in real life, deviations from this rule can often be made when a driver is a friend of the police officer, a celebrity, or has some other meaningful social attributes. In a narrow sense, particularism means that a given universal rule is violated due to the existence of personal relations between individuals (e.g., when a policeman lets a driver exceeding the speed limit go because he/she is a friend). In a broader sense, particularism leads to the violation of a rule because the object of judgment falls into some specific social category (e.g., a driver is female)

Universalism is not reducible to "rights" or the allocation of rewards, but implies the readiness to follow a specific rule, standard of behavior, or evaluation. According to T. Parsons (1937), universalism-particularism is treated as a "standard variable" characterizing various levels of action systems: cultures, institutions, and individual dispositions. Although empirical studies of universalism-particularism per se are relatively infrequent, they demonstrate the effects of particularism at various levels and in different domains.

Comparative studies conducted by F. Trompenaars and C. Hampden-Turner (2012) revealed important differences between countries in the degree to which people accept particularistic violations of the rules. Italian economists consider universalism-particularism as a latent factor underlying social capital, and use several proxies (e.g., trust or associational activity) to study the differences across countries and regions (DeBlasio, Scalise, \& Sestio, 2014). Ma and McLean Parks (2007) proposed a measure of particularism (in its narrow sense) at the individual level. Importantly, the concept is applicable to institutions as well as to individuals or cultures. For example, science is proposed to be an institution stressing universalism as its functionally necessary principle (Merton, 1957), but that does not mean that it always functions in universalistic manner (e.g., Fisman et al., 2018).

Universalism as a normative principle should be distinguished from universalism as a characteristic of a solution to a given situation provided by an individual, collective, or institutional actor. The two types can be called normative and practical universalism, respectively. Whereas the former indicates that an actor should apply the standard in one and the same way, the latter shows whether it is actually applied that way in a given situation. Basically, normative universalism is about intentions and normative orientations, and practical universalism is about actual outcomes. Beyond the question of whether and when universalism should be ex- 
pected and promoted in individual or collective behavior, there is a question of how and under what conditions it can actually be implemented.

Although the notion of universalism is very abstract and transcends disciplinary boundaries, it is directly related to social psychological notions of social bias, prejudice, and discrimination, which are the well-known effects of social categorization. Asymmetrical cognitive, attitudinal, and behavioral reactions to people with different social attributes are widely documented and seen as deeply rooted in an individual's psychological, cognitive, and neural make-up (e.g., Tajfel et al., 1971; Oakes, 2003; Van Bavel \& Cunningham, 2011; Kawakami, Amodio, \& Hugenberg, 2017; Liberman, Woodward, \& Kinzler, 2017).

As a psychological process, social categorization produces particularistic effects by making some groups preferable to others. Although such effects can differ and be moderated by many factors, there is a general tendency to prefer one's own group and make decisions in favor of it, i.e., ingroup favoritism - a tendency which is well-documented but remains controversial in terms of its theoretical explanation (Balliet, Wu, \& De Dreu, 2014; Greenwald \& Pettigrew, 2014; Everett, Faber, \& Crockett, 2015). As a cognitive process, social categorization determines the way people classify others into groups or categories, thus providing the lines along which the universalistic principle is violated.

A lot of effort has been spent in social psychology to identify the possible ways of reducing prejudice and discrimination, and thus promote fairer reactions and behaviors that correspond to the principle of universalism. Potential strategies include: reducing explicit prejudice via normative and institutional pressure; retraining and attitude change; increasing intergroup contacts; individuation; establishing common identity; and others, up to direct brain stimulation (Oscamp, 2008; Sellaro et al., 2015). What these strategies share is the intention to change long-term personal dispositions and improve intergroup relations. Setting aside the question of how realistic such hopes are, given the evolutionary logic underlying social categorization, there is also the question of how universalistic solutions can be obtained when eliminating biases and prejudice is impossible - probably a more typical and realistic situation.

One way that universalistic solutions in social situations may still be possible is through the cognitive complexity of most social situations and, particularly, multiple social categorizations (Stangor et al., 1992; Crisp \& Hewstone, 2006; Kang \& Bodenhausen, 2015). Multiple social categorization - i.e., classification of a target according to several distinct attributes - is considered a possible way to reduce prejudice, as it permits increased individuation and the counterbalancing effects of several single categorizations (Crisp \& Hewstone, 2007; Prati et al., 2016). When a situation involves different identities, the biases associated with them can overlap and partially compensate each other, and the very cognitive complexity of social targets can reduce bias. At the same time, such positive effects of multiple categorization are not inevitable. Hewstone et al. (2006, p.282) point to three possible factors reducing its effectiveness: the functional dominance of one social dimension; the correlation of categories; and group threat.

Most studies of multiple categorization focus on its ability to reduce prejudice in one specific social dimension, such as race or ethnicity. However, in many reallife situations, there is often no a priori information on which social dimension can 
become salient and influence actual decisions, such as in hiring a worker. Thus, it is not always clear how interventions targeting one specific social attribute will affect other available social categorizations. Furthermore, the ability to make universalistic decisions does not necessarily mean the reduction of prejudice toward a specific social group. That ability can be counterbalanced by other biases and prejudices or by the top-down control mechanisms (Cunningham et al., 2004).

Two other limitations of existing studies on multiple categorization are worth mentioning. First, most of them are conducted in Western societies, which are generally more universalistic and provide larger normative pressure on individuals to control their actual decisions. Second, by looking for strategies and interventions to reduce individual prejudices, psychological studies seem to ignore group-level solutions. Although social categorization underlies the psychology of intergroup relations as such (Oakes, 2003), the ability to find a universalistic solution to a social problem is not always due to changes in attitudes or behaviors. Social situations involving biases are not necessarily situations of intergroup relations, as in the case of making expert judgments on a politically sensitive issue. Thus, involving fundamental mechanisms of social categorization may extend the psychology of intergroup relations to find solutions to important social problems without improving intergroup relations as a necessary prerequisite.

Assuming that there can be different possible solutions in a situation involving multiple categories, one can expect that the aggregation of individual reactions at the group level can lead to more universalistic solutions. The present study addresses the afore-mentioned issues and investigates the possibility of universalistic solutions via different social biases counterbalancing each other, on either an individual or group level.

\section{Methods}

\section{Participants}

A socially heterogeneous sample of 300 individuals ( $61 \%$ female, mean age $=36.7$, $\mathrm{SD}=10.6)$ from Russia participated in a web-based study. Russia has a specific cultural profile favoring deviation from universalism. In a study by Trompenaars and Hampden-Turner (2012), Russia was classified as a highly particularistic culture according to all the three measures used by the researchers. In the GLOBE study, Russia showed a significant prevalence of ingroup collectivism over institutional collectivism, which can also indicate the preference for particularistic solutions (House et al., 2004). Additionally, in the Hofstede model, Russia was characterized as very high in the Power Distance dimension, which can be another source of particularism based on social status (Hofstede, Hofstede, \& Minkov, 2010). In sum, Russia is an example of a culture which is rather unfavorable for universalistic judgments and decisions.

The sample consisted of people from different regions and strata of Russian society. Participants were recruited via an online panel provided by an independent service provider, IOM Anketolog (https://anketolog.ru), and were paid for their participation. All the materials used in the study were moderated and approved by the service provider, and collected according to its rules. The mean time spent for filling the forms was 6:28 min, ranging, with one exception, from 2:18 to 32:13 min. 


\section{Procedure}

The design of the study was based on the forced-choice paradigm, which resembles the conjoint analysis frequently used by marketers to identify customers' preferences (Green, Krieger, \& Wind, 2001). Participants made a series of choices between two alternatives in a hypothetical situation. The task was described in the following way: "Imagine that mankind contacted a different civilization and someone has to represent and speak on behalf of all humans on Earth. Below you will be presented with several pairs of potential candidates to be such a representative. All candidates are represented by numbers and will be displayed in random order. Each of them is described by four characteristics $\langle\ldots\rangle$. Assuming that all the candidates' other characteristics which are important for this role are comparable, who do you think is most fit for this role?"

The four social descriptors included a candidate's country, gender, age, and sector of employment. The "Country" descriptor was used to manipulate two distinct social attributes: ingroup/outgroup $(\mathrm{I} / \mathrm{O})$ based on nationality or citizenship, and social status. I assumed that countries differ in how they are perceived by lay people, and that the hierarchical dimension is an essential part of this perception. I used two measures to identify the social status of a country: GDP and GDP per capita. The use of objective measures of economic power instead of subjective perception relies on the assumption that potential respondents have sufficient common knowledge to perceive the relative status of a country. I computed a list of all countries using GDP per capita as a multiplier for GDP. The countries were then classified into three categories:

- High-status outgroup (HSO): countries ranking 10 or more positions higher than Russia in the list (Germany, Japan, USA);

- Same-status outgroup (SSO): countries ranking within 10 positions of that of Russia (Argentina, Brazil, Turkey).

- Low-status outgroup (LSO): countries ranking 10 positions lower than Russia (Albania, Algeria, Azerbaijan, Cambodia, Colombia, Ghana, Jordan, Kenya, Moldova, Paraguay, Philippines, Sri Lanka, Vietnam).

In selecting specific countries, I also followed two additional rules. First, I tried to select countries which met the criteria mentioned above on both initial measures as well as a joint one. Second, I relied on common sense to make sure that the target country would be adequately perceived by a typical Russian participant, and avoided countries which could be perceived as too ambiguous (e.g., China), or too exotic. Finally, to minimize the possible effect of specific stereotypes associated with a particular country, each category was represented by several countries. For HSO and SSO, only a limited number of countries are available for inclusion, whereas many more countries fit the criteria for LSO.

Country and gender are the primary social dimensions compared in the study. As there are four different "Country" categories (Ingroup, HSO, SSO, LSO) and two gender categories (male $(\mathrm{M})$ - female $(\mathrm{F})$ ), 13 different types of choices involving at least one ingroup-outgroup difference can be analyzed: IM/IF; IM/HSOM; IM/HSOF; IM/SSOM; IM/SSOF; IM/LSOM; IM/LSOF; IF/HSOM; IF/HSOF; IF/ 
SSOM; IF/SSOF; IF/LSOM; and IF/LSOF. To directly test the effect of social status, four additional types of comparison have been included: HSOM/LSOM; HSOM/ HSOF; HSOF/LSOM; and HSOF/LSOF. Each of the 17 types of choice appeared twice in the study, in order to counterbalance the order of presentation (left-toright or up-or-down, depending on a user's device) and increase the reliability of the results. In sum, each participant made 34 choices among 68 different social profiles. An example of a choice situation is presented in Figure 1.

Make your choice

55

\begin{tabular}{|l|}
\hline \multicolumn{1}{|c|}{55} \\
\hline Country: Russia \\
Gender: Female \\
Age: $\mathbf{5 7}$ \\
Sector: Corporate \\
\hline
\end{tabular}

56

\begin{tabular}{|l|}
\hline \multicolumn{1}{|c|}{56} \\
\hline Country: Germany \\
Gender: Male \\
Age: $\mathbf{5 1}$ \\
Sector: Corporate
\end{tabular}

Figure 1. Example of a choice

Age and sector were two other social descriptors included in each profile to make the choice more complex and plausible. Both of them were included for each profile based on the randomization procedure, and were fixed for every participant. The age descriptor was randomly determined from a range between 35 and 70 years. The lower limit of 35 years old was chosen to make the choice situation more plausible (for example, 35 years is minimum age for becoming president in Russia). Candidates' sectors of employment were randomly selected from the three possible options: "corporate," "governmental/public," and "non-governmental."

The core assumption of the design was that not all social attributes in the profiles are relevant to the task. The instructions stressed that all relevant characteristics of candidates are equivalent, and that the focus on "the interests of all humans on Earth" can function as a kind of recategorization, which posits a higher-order common identity. It was assumed, that under the given conditions, not all the descriptors were relevant to the task and all the candidates should be treated equally, according to a universalistic approach (practical universalism). All systematic violations then should be treated as biases (particularistic solutions).

\section{Measures}

According to the key assumption of the study, the universalistic solution for a given situation implies equal chances for each candidate to be selected for the role. Unlike normative universalism, which is related to personal predispositions, practical universalism involves actual outcomes of situations involving social categorization. Thus, a simple measure of universalism is the absence of systematic bias across multiple choices. In statistical terms, this means that the universalistic solution is equivalent to the null hypothesis (none of the social descriptors affect the prospects for each alternative). The present design allows us to analyze the effects of social categorization on both a group and individual level. 


\section{Group-Level Measures}

According to the null hypothesis (i.e., universalistic solution), all candidates have equal chances to be selected by the participants. If the group as a whole prefers one option in each pair, it should be treated as a measure of bias, i.e., particularism. The larger the difference between the group solution and the choice by chance, the more particularistic it is.

First, each of the 34 choices can be analyzed in terms of acceptance or rejection of the null hypothesis using a one-sample binomial test. Second, the frequencies of choices can be combined to measure the preference for each specific social category of interest.

Every choice situation can be described in terms of a specific social preference: primary ingroup over outgroup, primary ingroup over HSO, male over female, etc. If a group as a whole behaves in a universalistic manner, the expected frequency of each chosen alternative is $50 \%$ (of all the situations where such a choice is possible). Any deviations from this number averaged across all the relevant social comparisons is a simple and direct measure of particularism.

\section{Individual-Level Measures}

Individual preferences for a specific social group can also be measured directly, by computing all choices in favor of each category of interest (primary and gender ingroup, high or low status, etc.). The degree of favoritism (particularism), i.e., the preference for a particular category for each dimension, is measured as

$$
P_{\text {cat }}=\text { Number of } c a t \text { choices }-\mathrm{n} / 2 \text {, }
$$

where cat is a specific social category of interest (ingroup, high status, male, etc.), and $\mathrm{n}$ is the total number of choices when this category has an alternative (e.g., I vs $\mathrm{O}$, HSO vs LSO, etc.)

Here, $P_{\text {cat }}=0$ is equivalent to a universalistic solution, and the more it deviates from 0 , the larger is a participant's specific social preference. Positive numbers mean positive discrimination in favor of $c a t$, whereas negative numbers mean negative discrimination. Individual strategies can be identified according to preferences for specific social categories.

\section{Results}

\section{Group-Level Results}

The average response frequencies across all 34 choices diverged from the universalistic solution by 14.78 percentage points. This means that, on average, one of two alternatives has been chosen $64.78 \%$ of the time, and the other, $35.22 \%$ of the time. This number is the direct overall measure of particularism in a given situation.

To understand the main sources of such a deviation from a universalistic solution, similar measures were calculated for each social attribute (Table 1). 
Table 1

Mean violations from universalistic solutions for key social categories across all the choices in which they were compared

\begin{tabular}{lcc}
\hline Contrast (bias type) & $\begin{array}{c}\text { Deviation from the expected dis- } \\
\text { tribution 'by chance' across all the } \\
\text { relevant choices, percentage points }\end{array}$ & $\begin{array}{c}\text { Mean absolute value of all } \\
\text { the deviations across relevant } \\
\text { choices, percentage points }\end{array}$ \\
\hline I $>$ O & 13.12 & 14.32 \\
I $>$ HSO & 6.53 & 8.80 \\
I $>$ SSO & 13.29 & 14.61 \\
I $>$ LSO & 19.54 & 19.54 \\
HSO $>$ LSO & 13.91 & 16.34 \\
M $>$ F & 3.63 & 15.38 \\
Gender I $>$ O & 3.79 & 15.38 \\
Younger $>$ Older & 1.65 & 14.78 \\
Corporate sector bias & -0.15 & 16.45 \\
Governmental sector bias & 0 & 17.33 \\
NGO sector bias & 0.28 & 14.03 \\
\hline
\end{tabular}

Notes: $I=$ ingroup; $O=$ outgroup; $H S O=$ high-status outgroup; $S S O=$ same-status outgroup; $L S O=$ lowstatus outgroup; $M=$ male; $F=$ female. Positive meanings of the "deviation" variable mean discrimination in favor of the first element of the contrasted categories; negative numbers show bias for the second one. The absolute mean value of deviation indicates how biased the choices, included in the computation, are on average.

As shown in Table 1, primary ingroup-outgroup distinction based on affiliation with a country, and perceived social status were the two main sources of particularism in the sample. The participants, on average, preferred candidates from their own country and candidates of higher status, even though the task implied taking the perspective of all humankind. The fact that the ingroup preference increased gradually when contrasted with high-, same- and low-status outgroups, confirmed that the method of country classification by status was effective.

The group also demonstrated a moderate preference for male candidates and, at the same time, for a gender ingroup, along with some preference for younger candidates. There was no effect of the employment sector.

The fact that at least three social attributes contributed to the group's particularism implies that, under some conditions, particular biases can counterbalance each other and produce a universalistic solution. One-sample binomial test $(\alpha=0.5)$ allowed me to estimate the group's solution for each of 34 choices. In 26 cases, the null hypothesis was rejected, i.e., the group decisions were particularistic. However, the remaining eight cases could be treated as universalistic. Table 2 describes key information about these choices. 
Table 2

Key social attributes of universalistic choices

\begin{tabular}{lcccl}
\hline \multicolumn{1}{c}{ Main contrast } & $\begin{array}{c}\text { Age difference, } \\
\text { years }\end{array}$ & $\begin{array}{c}\text { Younger social } \\
\text { group }\end{array}$ & $\begin{array}{c}\text { Sector difference } \\
(1-\text {-yes, } 0-\text { no })\end{array}$ & $\begin{array}{c}\text { Outgroup } \\
\text { country }\end{array}$ \\
\hline IF-HSOM & 6 & HSOM & 0 & Germany \\
IF-HSOM & 13 & HSOM & 0 & Japan \\
IF-HSOF & 20 & HSOF & 1 & Germany \\
IF-HSOF & 9 & HSOF & 0 & USA \\
IM-HSOM & 14 & HSOM & 0 & Germany \\
IF-SSOF & 33 & SSOF & 1 & Brazil \\
LSOF-HSOF & 9 & LSOF & 1 & Moldova-USA \\
LSOM-HSOM & 13 & LSOM & 0 & Jordan-USA \\
\hline
\end{tabular}

Looking at these universalistic cases, one might suggest that the country-based ingroup favoritism can be counterbalanced by the other candidates' higher status, younger age, and probably gender. All the other choices are particularistic. Interestingly, the two most particularistic choices, with more than 30 p.p. deviation from the choice "by chance," did not involve the primary ingroup and were based on status dimension ( $\mathrm{HSOF}>\mathrm{LSOF}$ and $\mathrm{HSOF}>\mathrm{LSOM}$ ).

\section{Individual-Level Results}

According to the study design, the participants faced a decision point which was relatively complex and cognitively challenging. The necessary trade-off between several social attributes might lead to an explicit or implicit choice of a particular strategy, or a rule (set of rules) for deciding each situation. Analysis of the frequency distributions for each social attributes showed that the participants significantly differed in their biases (Figure 2).

The participants demonstrated very diverse biases in terms of both direction and strength. In most cases, the distribution of preferences was close to normal, including preferences regarding the sector of employment (not depicted). Most notable was the asymmetrical distribution for the main ingroup-outgroup contrast, where a significant portion of female participants always chose their country whenever it was compared to other countries. However, two-sample t-tests $(\alpha=0.05)$ showed there were no statistically significant differences between male and female participants for all contrasts except the preference for their gender ingroup. Here, the difference was significant: $\mathrm{t}$-criterion $=5.15(\mathrm{df}=298, \mathrm{P}<0.001)$. This means that, although both male and female participants tended to prefer their gender ingroup (mean preference is 1.81 and 0.03 , respectively), women more frequently preferred their gender outgroup. 
a)

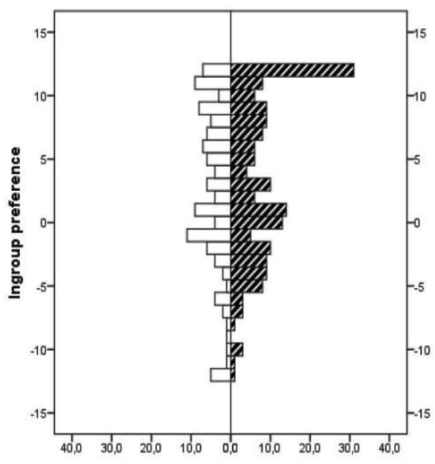

d)

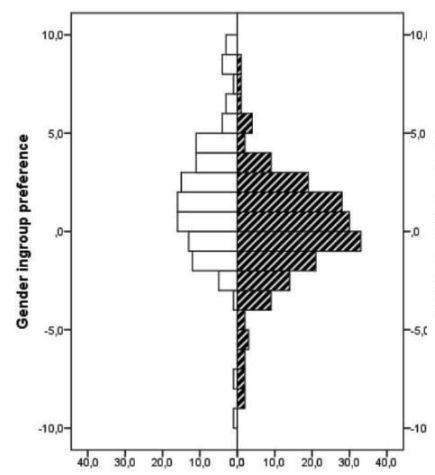

b)

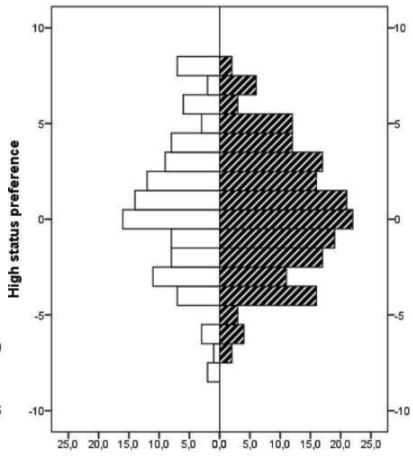

e)

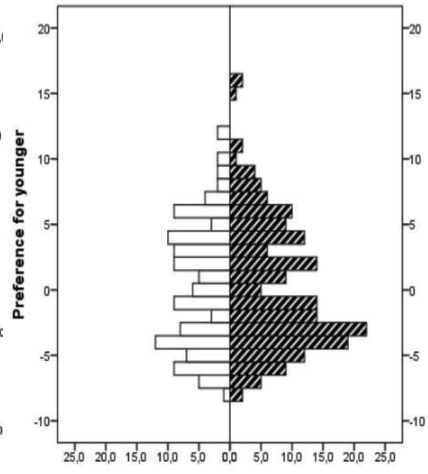

c)

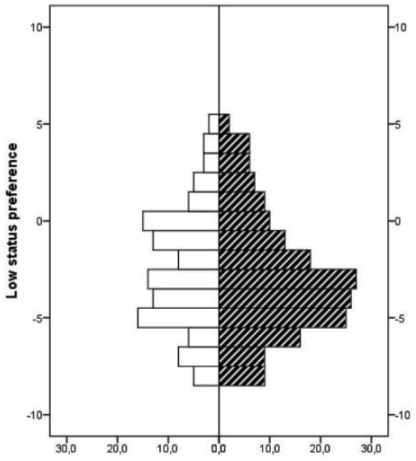

$\square$ Male participants

Female participants

Figure 2. The distribution of individual preferences for each social attribute $\left(\mathrm{P}_{\text {cat }}\right)$, male and female participants.

Notes. Axis $X=$ frequency; axis $Y=$ Preference for: $a)$ country ingroup $(I>O) ; b)$ high status $(H S O>I$ and $H S O>L S O)$; c) low status (LSO $>I$ and LSO $>H S O$ ); d) Gender ingroup (gender I > gender O); e) younger candidates (younger $>$ older). Left panel - male participants, right panel - female participants

The correlational analysis showed how different types of bias were related to each other (see Table 3).

Table 3

Coefficients of correlations for key types of bias

\begin{tabular}{lcccccc}
\hline \multicolumn{1}{c}{ Preference } & $\begin{array}{c}\text { Country } \\
\text { ingroup }\end{array}$ & High status & Low Status & Male & $\begin{array}{c}\text { Gender } \\
\text { ingroup }\end{array}$ & Younger \\
\hline Country ingroup & - & $-0.68^{* *}$ & $-0.63^{* *}$ & -0.01 & 0.08 & $-0.76^{* *}$ \\
High status & & - & -0.10 & 0.08 & -0.11 & $0.39^{* *}$ \\
Low Status & & & - & -0.04 & 0.00 & $0.65^{* *}$ \\
Male & & & & - & 0.03 & 0.02 \\
Gender ingroup & & & & & - & -0.09 \\
\hline
\end{tabular}

Note. ${ }^{* *}$ correlations significant at level 0.01 . 
Country ingroup preference negatively correlated with both status measures. More interestingly, high- and low-status preferences were not correlated with each other in the current research. Additionally, age-based preference for younger candidates was negatively correlated with country ingroup bias, and positively with both status biases. Age bias was not correlated with the age of participants. Gender was not associated with any other type of bias.

To better understand the nature of individual strategies followed by participants, their values of all types of bias $\left(P_{c a t}\right)$ were analyzed and combined. For this purpose, every preference scale was transformed into a universal ordinal bias scale ranging from (-2) to (2). Correspondingly, all participants were classified as having high $(-2 ; 2)$ moderate $(-1,1)$ or low $(0)$ bias in every social dimension. The percentages of those having a high bias in at least one social dimension, are shown in Table 4.

Table 4

Percentage of participants with high bias in one social dimension

\begin{tabular}{lc}
\hline \multicolumn{1}{c}{ High bias type } & \% of all participants \\
\hline Ingroup preference & 31.7 \\
Outgroup preference & 5.0 \\
High-status preference & 8.7 \\
High-status aversion & 4.0 \\
Low-status preference & - \\
Low-status aversion & 17.7 \\
Gender ingroup preference & 4.7 \\
Gender outgroup preference & 2.7 \\
Preference for younger & 2.3 \\
Preference for older & - \\
\hline
\end{tabular}

Most of the other participants pursued strategies based on moderate bias types in various combinations. This means that the current combination of social categories does not lead to a counterbalancing effect and practical universalism in most cases. There was only a small portion of participants (2.0\%) with low bias in all social dimensions, i.e., those who pursued the most universalistic strategies. In sum, the data confirmed a variety of bias types which underlie individual strategies for choosing the best alternative at every decision point.

\section{Discussion}

The present study interprets the effect of multiple categorization on perception and behavior in terms of practical (rather than normative) universalism, i.e., the actual outcomes of situations involving several systems of social categorization not relevant to the nature of a decision. Although the hypothetical situation used in the current design may seem too artificial, it emulates the logic of many real-life 
situations. Examples include choosing among candidates for a job position or a role (when only job-related characteristics should be evaluated); assessment of employees' or students' achievements; evaluating judgments on policy issues; selecting which scientific articles on a topic to read and rely upon; and many others. There are many situations where ignoring social attributes that are not relevant for the task are crucial and expected.

This study showed that a sample of Russian participants has a strong tendency to make particularistic choices based on social attributes. The fact that the null hypothesis used to measure the lack of bias was rejected in the majority of choices, indirectly validates the possibility of using this approach in the study of multiple categorization. However, precautions should be noted before concluding that the statistical lack of choice preference is actually the result of social cognition mechanisms.

Deviation from universalism occurred despite the fact that the situation was not motivationally significant for individuals, and that a higher-order common identity was salient. This may be not very surprising since Russia has been found to be among the most particularistic and status-sensitive countries (Trompenaars \& Hampden-Turner, 2012; Hofstede, Hofstede, \& Minkov, 2010). Even the results regarding gender bias are consistent with Russia's cultural profile. Although there is an identifiable male preference in the current study, its effect is relatively small. Both country-level and cross-cultural studies show that, although there is some gender inequality in favor of men in Russia, this inequality is not very high, and egalitarian views in gender relations are widely accepted (Inglehart et al., 2014; Pew Research Center, 2019).

What is more interesting is the diversity of the participants' biased responses and their strategic nature. Whereas a particularistic normative system makes actual deviations from universalism more acceptable, it does not specify the nature of the social attributes according to which such deviations can be aligned. Discussions in the field of multiple or cross-categorization, make different predictions on how individuals should respond to a situation when the information about several social dimensions is available. According to Nicholas, de la Fuente, \& Fiske (2017), there are two general theoretical models which predict that when a person faces two or more competing social dimensions of a target, the effect on their social attitudes can be based either on combining biases and preferences for each category (algebraic models), or on the complete dominance of one specific category (nonalgebraic models). The present study shows that these alternative models are not necessarily contradictory and can be considered as different cognitive mechanisms involved in actual decision-making.

Deciding on a complex social situation involving lots of objects and various social dimensions can be better explained as a matter of strategic choice. Such a strategy, either implicitly or explicitly, includes making the decision as to which information should be prioritized, and how it should be combined with other information. The individuals making decisions in the present study differed in their strategies and followed different rules, probably reflecting differences in personal dispositional traits.

Some of them always chose their own (country) group, others always chose outgroups, some always chose objects of higher status, others always rejected them, 
some only chose a specific gender, etc. Many others used more complicated strategies which combined various social information in a more balanced way. There is evidence that specific stereotypes of a particular group can contribute to a decision, as well as abstract relational structures (e.g., ingroup-outgroup, high-low status). Finally, a small portion of participants demonstrated low bias across all the dimensions.

This variety of individual strategies challenges the view that multiple social categorization can necessarily reduce particular bias or prejudice (Prati et al., 2016; Kang \& Bodenhausen, 2015). People seldom have access to only one type of social information. Rather, they typically face the complexity of social information about others, and then seek to reduce it and develop specific rules to make decisions and evaluations faster and easier.

The question is how the available social information is prioritized and structured. The current research showed that, in the absence of contextual or motivational factors, two social dimensions are especially important to a person making the choice. A strong negative correlation between ingroup affiliation and social status suggests there is a significant trade-off between these two social attributes. Taking into account that affiliation and social hierarchy are two qualitatively distinct types of social relations (Fiske, 1992; Gilboa-Schechtman \& Shachar-Lavie, 2013), one can suggest that this trade-off is in fact between the two cognitive mechanisms which support processing affiliation- and hierarchy-related social information. A similar conclusion has recently been made regarding the neural processing of race and social status by Mattan et al., (2018).

Gender and age are two other social dimensions contributing to deviations from universalism. Importantly, the results of the study indicate that gender categorization is separate from other types of categorization. This corresponds to some previous findings which showed that gender is a specific social category independent of other types of social knowledge (Sidanius et al., 2004).

Turning back to the key question of the possibility of, and conditions for, universalistic solutions in multiple categorization situations, the findings of the present study are twofold. On the individual level, there is little evidence that different biases can effectively counterbalance each other. Rather, individuals facing a series of identical situations, tend to pursue a specific type of bias resulting in particularistic solutions. However, evidence of a trade-off between different social categorizations indicates that some balancing effects of alternative biases exist, and may contribute to partial inhibition of particularistic choices. Further research is needed to better understand the conditions and situations where such a balance can be achieved in individual judgments and decisions.

At the same time, the diversity of biases and individual strategies opens interesting opportunities for the avoidance of particularism at the group level. Although there was a group of participants who pursued universalistic strategies, it was relatively small. However, the fact that participants were biased in different ways means that under some conditions, a group of people with counterbalancing strategies of social categorization can produce universalistic solutions as a whole. The present study included examples of such situations, in which specific combinations of social attributes resulted in equal distribution of choices. This means that, for a 
given group of people, there exists a combination of salient social attributes which induces biases to counterbalance each other.

To see the practical significance of this information, consider the situation when the task is to choose a candidate for a specific role, or evaluate someone's achievement, judgment, or behavior. From the present research, we might conclude that for the situations described in Table 2, the group we studied could make unbiased judgments and decisions.

Alternatively, we can conclude that for any given situation involving multiple categorization, it is possible to find a group of biased individuals who can jointly produce a fair evaluation, judgment, or decision. One can imagine a hypothetical mechanism for assembling a collective body which is able to make universalistic decisions irrespective of both individual biases and institutional solutions for such purposes as, perhaps, a blind review in science. Juries, expert boards, or commissions are examples of such collective actors, which are supposed to be able to produce unbiased judgments and/or solutions. Importantly, even particularistic and non-democratic societies need the ability to produce unbiased solutions - for example, in making expert judgments or evidence-based decisions.

Whether the pattern of reactions in our sample of Russian participants is generalizable to other types of situations and in other societies is a matter for further research. A direct comparison with samples from various cultures would be especially important. In particular, one might expect that participants from more universalistic and egalitarian cultures will generally pursue more universalistic strategies in their choices.

The situation, however, can be more complicated. Social categorization is an objective source of biases and prejudices, but in universalist cultures, greater normative pressures can counterbalance them in behavioral outcomes. However, the very complexity of multitude social choices provided by the current design makes it difficult to balance all the possible biases arising from social categorization. The attempt to compensate for implicit biases and prejudice may well result in positive discrimination, which also violates universalistic rules and which was found in the present research.

In support of this view, a recent study by Kteily, Sheehy-Skeffington, and Ho (2017) found that people with both egalitarian and anti-egalitarian views differ in their perception of inequality. It is thus possible to suppose that in more universalist cultures, a greater share of biases toward outgroup and low-status groups can be expected due to the cognitive inability to perfectly adjust control over one's biases. Thus, group-based solutions exploiting the diversity of individual social biases can be a promising new way to practical universalism in various social and cultural settings.

\section{Conclusion}

Social categorization is the source of asymmetrical cognitive, attitudinal, and behavioral reactions which deviate from universalistic norms and inhibit the ability of people to act in a non-biased, unprejudiced, and non-discriminatory way. When faced with complex social information and multiple categorization, individuals have to develop a strategy to deal with this complexity. The present study, con- 
ducted in a country with a particularistic value system, explores the possibility that alternative social categorizations can produce biases which counterbalance each other in a given situation, without changing long-term personal attitudes toward specific social groups. The study allows us to suggest that multiple social categorization can indeed produce alternative biases, which may act in opposite directions.

In particular, the effect of country ingroup favoritism can potentially be diminished by high-status preference. However, the counterbalancing effect of this 'conflict of biases"' in practical behavior is limited. When an individual faces a series of similar situations involving multiple categorization, he or she tends to develop a strategy, implicitly or explicitly, which mainly relies upon one specific type of bias. Strategies of country-ingroup preference and low-status aversion are the two most frequent.

At the same time, the diversity of biases and strategies adopted by individuals can allow the achievement of universalistic solutions at the group level. The present study sheds light on the conditions under which universalistic solutions are possible even in particularistic societies. A group of biased individuals can behave in an unbiased way, when the social attributes of the targets, or the individuals' own social biases, counterbalance each other. This finding shows the possibility of social designs exploiting the cognitive and psychological mechanisms of social categorization, which allow the creation of reliable social systems from unreliable elements.

\section{Limitations}

There are several limitations of the current study which can affect its significance and generalizability. First, although the sample used was more representative than in many psychological studies, it still lacked the representativeness of a good sociological study. At the same time, the idea behind the present study was that any heterogeneous group can, in principle, produce unbiased solutions.

Second, the study design focused only on two main social descriptors: country and gender. The data regarding the two other descriptors was less reliable.

Third, the social profiles of the alternatives had a fixed structure and order in which the social descriptors were organized. This could have affected the relative importance of various social dimensions.

Fourth, the current design and data analysis techniques did not allow us to differentiate between universalistic strategies based on rational decisions, and those which originated from the participants' dishonesty and chaotic responses. Additionally, the use of the "null hypothesis" interpretation of practical universalism can be problematic and result from factors which are not controlled in the study. Finally, the study was conducted in only one country, and assessing the role of cultural values requires additional studies.

\section{Acknowledgements}

I thank Sergio Chaigneau and one anonymous reviewer for useful suggestions and stimulating criticism on an earlier version of this article. 


\section{References}

Balliet, D., Wu, J., \& De Dreu, C.K.W. (2014). Ingroup favoritism in cooperation: A meta-analysis. Psychological Bulletin, 140, 1556-1581. https://doi.org/10.1037/a0037737

Everett, J.A.C., Faber, N.S., \& Crockett, M. (2015). Preferences and beliefs in ingroup favoritism. Frontiers in Behavioral Neuroscience, 9. https://doi.org/10.3389/fnbeh.2015.00015.

Crisp, R.J., \& Hewstone, M. (Eds.) (2006). Multiple social categorization: Processes, Models, and Applications. Hove; New York: Psychology Press. https://doi.org/10.4324/9780203969229

Crisp, R.J., \& Hewstone, M. (2007). Multiple social categorization. Advances in Experimental Social Psychology, 39, 163-254. https://doi.org/10.1016/S0065-2601(06)39004-1

Cunningham, W.A., Johnson, M. K., Raye, C.L., Gatenby, J.C., Gore, J.C., \& Banaji, M.R. (2004). Separable neural components in the processing of Black and White faces. Psychological Science, 15, 806-813. https://doi.org/10.1111/j.0956-7976.2004.00760.x

De Blasio, G., Scalise, D. \& Sestio, P. (2014). Universalism vs Particularism: a round trip from sociology to economics. Bank of Italy Occasional Paper, 212. https://doi.org/10.2139/ssrn.2419937

Fiske, A.P. (1992). The four elementary forms of sociality: Framework for a unified theory of social relations. Psychological Review, 99, 689-723. https://doi.org/10.1037/0033-295X.99.4.689

Fisman, R., Shi, J., Wang, Y., \& Xu, R. (2018). Social ties and favoritism in Chinese Science. Journal of Political Economy, 126, 1134-1171. https://doi.org/10.1086/697086

Gilboa-Schechtman, E., \& Shachar-Lavie, I. (2013). More than a face: a unified theoretical perspective on nonverbal social cue processing in social anxiety. Frontiers in Human Neuroscience 7, article 904. https://doi.org/10.3389/fnhum.2013.00904

Green, P.E., Krieger, A.M., \& Wind, Y. (2001). Thirty Years of Conjoint Analysis: Reflections and Prospects. INFORMS Journal of Applied Analytics, 31(3_supplement), S56-S73. https://doi. org/10.1287/inte.31.3s.56.9676

Greenwald, A.G., \& Pettigrew, T.F. (2014). With malice toward none and charity for some: Ingroup favoritism enables discrimination. American Psychologist, 69(7), 669-684. https://doi.org/10.1037/ a0036056

Hewstone, M., Turner, R.N., Kenworthy, J.B., \& Crisp, R.J. (2006). Multiple social categorization: Integrative themes and future research priorities. In Crisp, R.J., \& Hewstone, M. (Eds.), Multiple social categorization: Processes, Models, and Applications (pp. 271-310). Hove; New York: Psychology Press.

Hofstede, G., Hofstede, G.J., \& Minkov, M. (2010). Cultures and Organizations: Software of the Mind. New York: McGraw-Hill.

House, R.J., Hanges, P.J., Javidan, M., Dorfman, P.W., \& Gupta, V. (Eds.). (2004). Culture, leadership, and organizations: The GLOBE study of 62 societies. Thousand Oaks: Sage publications.

Inglehart, R., Haerpfer, C., Moreno, A., Welzel, C., Kizilova, K., Diez-Medrano, J., ... Puranen, B. (Eds.). (2014). World Values Survey: Round Six - Country-Pooled Datafile Version. Retrieved from: http://www.worldvaluessurvey.org/WVSDocumentationWV6.jsp. Madrid: JD Systems Institute

Kang, S.K., \& Bodenhausen, G.V. (2015). Multiple identities in social perception and interaction: challenges and opportunities. Annual Review of Psychology, 66, 547-574. https://doi.org/10.1146/ annurev-psych-010814-015025

Kawakami, K., Amodio, D.M., \& Hugenberg, K. (2017). Intergroup perception and cognition: An integrative framework for understanding the causes and consequences of social categorization. Advances in Experimental Social Psychology, 55, 1-80. https://doi.org/ 10.1016/bs.aesp.2016.10.001

Kteily, N.S., Sheehy-Skeffington, J., \& Ho, A.K. (2017). Hierarchy in the eye of the beholder: (anti-) egalitarianism shapes perceived levels of social inequality. Journal of Personality and Social Psychology, 112, 136-159. https://doi.org/10.1037/pspp0000097

Liberman, Z., Woodward, A.L., \& Kinzler, K.D. (2017). The origins of social categorization. Trends in Cognitive Science, 7, 556-568. https://doi.org/10.1016/j.tics.2017.04.004

Ma, L., \& McLean Parks, J. (2007). Rules or Relationships: Scale Development of Universalism versus Particularism as Individual Values. In IACM 2007 Meetings Paper. https://doi.org/10.2139/ ssrn. 1111663 
Mattan, B.D., Wei, K.Y., Cloutier, J., \& Kubota, J.T. (2018). The social neuroscience of race-based and status-based prejudice. Current Opinion in Psychology, 24, 27-34. https://doi.org/10.1016/j. copsyc.2018.04.010

Nicolas, G., de la Fuente, M., \& Fiske, S.T. (2017). Mind the overlap in multiple categorization: A review of crossed categorization, intersectionality, and multiracial perception. Group Processes \& Intergroup Relations, 20(5), 621-631. https://doi.org/10.1177/1368430217708862

Oakes, P. (2003). The root of all evil in intergroup relations? Unearthing the categorization process. In Brown, R. \& Gaertner, S.L. (Eds.), Blackwell handbook of social psychology: Intergroup processes (pp. 3-22). Malden: Blackwell Publishers.

Oscamp, S. (Ed.) (2008). Reducing Prejudice and Discrimination. Hove: Psychology Press.

Parsons, T. (1937). The Structure of Social Action. New York: McGraw-Hill.

Pew Research Center (2019). A Changing World: Global Views on Diversity, Gender Equality, Family Life and the Importance of Religion. Retrieved from: https://www.pewresearch.org/global/ wp-content/uploads/sites/2/2019/04/Pew-Research-Center_Global-Views-of-CulturalChange_2019-04-22.pdf

Prati, F., Crisp, R.J., Meleady, R., \& Rubini, M. (2016). Humanizing outgroups through multiple categorization: The roles of individuation and threat. Personality and Social Psychology Bulletin, 42(4), 526-539. https://doi.org/10.1177/0146167216636624

Sellaro, R., Derks, B., Nitsche, M.A., et al. (2015). Reducing Prejudice Through Brain Stimulation. Brain Stimulation, 8, 891-897. https://doi.org/10.1016/j.brs.2015.04.003

Sidanius, J., Pratto, F., van Laar, C., \& Levin, S. (2004) Social dominance theory: Its agenda and method. Political Psychology, 25(6), 845-880. https://doi.org/10.1111/j.1467-9221.2004.00401.x

Stangor, C., Lynch, L., Duan, C., \& Glass, B. (1992). Categorization of individuals on the basis of multiple social features. Journal of Personality and Social Psychology, 62, 207-218. https://doi. org/10.1037/0022-3514.62.2.207

Tajfel, H., Billig, M.G., Bundy, R.P., and Flament, C. (1971). Social categorization and intergroup behaviour. European Journal of Social Psychology, 1(2), 149-178. https://doi.org/10.1002/ ejsp. 2420010202

Trompenaars, F., \& Hampden-Turner, Ch. (2012). Riding the Waves of Culture. Understanding Diversity in Global Business. $3^{\text {rd }}$ ed. London: Nicholas Brealey Publishing

Van Bavel. J. \& Cunningham, W.A. (2011). A social neuroscience approach to self and social categorization: A new look at an old issue. European Review of Social Psychology, 21, 237-284. https:// doi.org/10.1080/10463283.2010.543314

Original manuscript received August 26, 2019

Revised manuscript accepted August 7, 2020

First published online September 15, 2020

To cite this article: Shkurko, A.V. (2020). Practical Universalism and Multiple Social Categorization: Can Different Social Biases Counterbalance Each Other? Psychology in Russia: State of the Art, 13(3), 96-112. DOI: 10.11621/pir.2020.0307 